\title{
Modelling the Synchronisation Control for a Hydro Power Controller
}

\author{
Jonatan Hellborg Tonje Tollefsen Khemraj Bhusal Dietmar Winkler \\ Department of Electrical Engineering, IT and Cybernetics, University of South-Eastern Norway, Porsgrunn, Norway \\ (contact: dietmar.winkler@usn.no)
}

\begin{abstract}
This paper presents the modelling of a synchronisation control as used inside a typical hydro power controller for small hydro power plants. It was built using the open-source modelling language Modelica by use of the Modelica Standard Library (Modelica Association 2019), the OpenIPSL (ALSETLab et al. 2018) and the OpenHPL (TMCC 2019).

The resulting model allows for both transient and longterm simulations for the complete hydro power system with the main functions available and working. This includes water-level control, frequency control, voltage control with a power factor control and the synchronisation sequence.
\end{abstract}

Keywords: Modelica, hydro power, synchronisation, hydro power controller

\section{Introduction}

As the importance of the transition to a carbon-neutral society increases, so does the need for tools that accelerates this problem. Because Norway is a country with great possibilities for hydro power, both large and small, there exists an inherent need for tools that allows for accurate simulations of existing plants but also the ability to simulate plants before they are built.

Norway holds a special place in the European electrical power grid because of the large usage of hydro power. Not only is $98 \%$ of Norway's electrical power hydro (Petroleum and Energy 2016), but it also accounts for an incredible $50 \%$ of Europe's reservoir capacity. (Statkraft 2020)

As more focus is given to the transition to a completely carbon-neutral society, the development of small scale hydro power has seen an increase, and with this increase comes the need for better tools that allow for better and more accurate simulations. These tools and the knowledge required to properly utilise them will help engineers and designers to find potential design flaws earlier in the design process, before the turbine is deployed. This becomes more and more important as more smaller companies (or even municipalities) decide on building small hydro power systems.

\section{The Hydro Power Controller}

A typical hydro power controller is made up of several components that realise different key functionalities of a hydro power station. They can be grouped into two main groups:

- The turbine governing functions act on the hydro turbine by providing the control signal for the guide vanes or nozzle opening (depending on the turbine type).

- The generator governing functions provide the signal for the excitation system in order to control the voltage and/or power-factor.

The controller implemented as part of this work is a typical hydro power controller for small hydro power stations (with a power rating between $1 \mathrm{MW}$ and $10 \mathrm{MW}$ ). It provides the following functionalities:

1. Turbine governing

(a) Speed control

(b) Water-level control

2. Generator governing

(a) Voltage regulation

(b) Power-factor control

A typical hydro power controller contains several additional functions which are not focus of this work.

\subsection{Turbine Governing}

The Turbine governor is the main controller of the hydraulic turbine. The governor regulates the turbinegenerator speed by controlling the flow rate of the water into the turbine. A higher water flow through the turbine means more torque is applied to the turbine runner which results in a higher applied mechanical power. Depending on the power balance the speed of the turbine and the directly connected generator will either increase or decrease.

The system of governing basically consists of a control section and a (mechanic/hydraulic) actuation section, see Figure 1. 


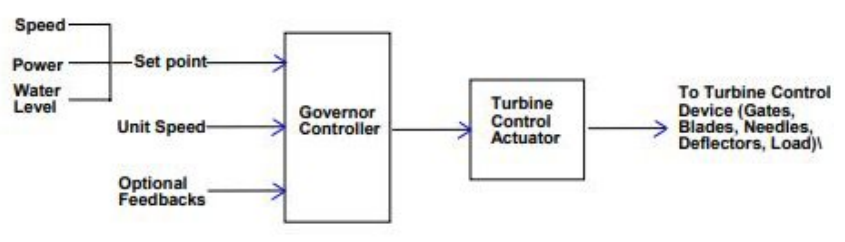

Figure 1. Basic Governor Control System (OD Thapar 2008)

\subsubsection{Speed Control}

The speed control is responsible for keeping the speed at which the turbine is spinning as close as possible to a given set-point so that the generator, which is directly coupled with the turbine, can produce the correct frequency. The controller has four different operation modes:

- Disconnected

- Normal

- Unstable

- Isolated

Each of these modes uses different sets of control parameters to suite the different nature of the electrical load experienced by the generator.

\subsubsection{Water-Level Control}

The main purpose of the water-level control in the hydro power system is to maintain an optimal water-level at the intake. This kind of control is especially common for small hydro power stations with small intake reservoirs (e.g., run-of-river types) where the varying precipitation can cause quite a change in the water-level. Operators use different strategies for their hydro power plants which means that the water-level controller needs to accommodate a different water-flow to guide vane opening characteristic.

A PI controller usually controls the output signal for the guide vanes for bringing the process variable (i.e., the water-level) to the desired value. If there is increment of the water-level at the intake, the guide vanes opening of the turbine should then respond by increasing the opening of the guide vanes so that the desired set point is reached.

If the water-level is below any specific level, the waterlevel controller will stop the turbine. This is important to avoid running out of water which then would lead to air getting into the waterway.

\subsection{Generator Governing}

\subsubsection{Voltage Regulation}

When a varying load is subjected to a generator then voltage at the armature terminals will vary to a certain extent. The amount of this variation determines the regulation of the machine. The voltage regulation, $V R$, of a generator can be defined as the change in terminal voltage from full load, $V_{f l}$, to no load, $V_{n l}$, expressed as a percentage of full load volts, when the speed and excitation field current are held constant (Pterra Consulting 2021).

$$
V R=\frac{V_{n l}-V_{f l}}{V_{f l}} \cdot 100 \%
$$

The full-load voltage is the terminal voltage when full load current is drawn. The no-load voltage is the one when zero current is drawn from the supply, i.e., open circuit terminal voltage.

The purpose of a voltage regulator is to keep the voltage within the prescribed range.

The voltage regulation is normally achieved by an excitation system which usually consists of an automatic voltage regulator (AVR), an exciter, measuring elements, a power system stabiliser (PSS) and limitation and protection units, see Figure 2.

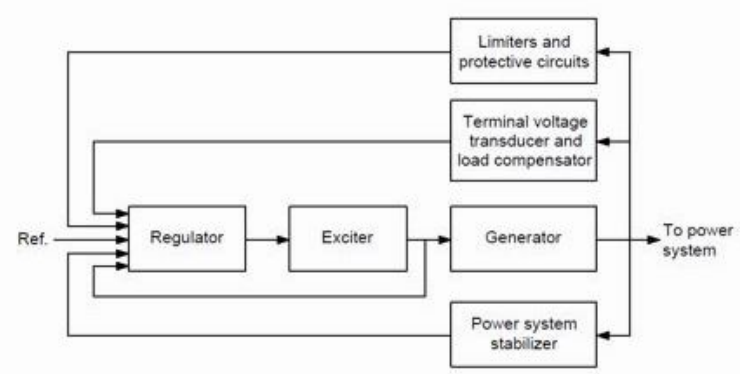

Figure 2. Block diagram of an excitation system of a synchronous generator (Pterra Consulting 2021)

The current and voltage produced by the exciter is usually controlled by the AVR. In case of sudden disturbances, there may be negative influences on the damping of power swings. For this, a supplementary control loop, the PSS, is introduced. The PSS produces an additional signal which is injected into control loop in order to compensate any voltage oscillations.

\subsubsection{Power Factor Regulator}

In general, an excitation system used in synchronous generators is expected to aid in the regulation of the system grid voltage. However, for small hydro power plants, the voltage regulation from the excitation system will not have a large impact on the system grid voltage. Therefore, operators of small hydro power plants often utilise a power factor regulator to regulate the excitation voltage so that the generator operates at power factor 1 . The power factor regulator is connected to the AVR as an outer loop control that takes direct control of the excitation voltage. It is important to note that a power factor regulator may not be suitable to handle voltage instability that may occur from faults, and therefore should not act as a replacement for the AVR entirely (IEEE 2016).

\subsection{Synchronisation}

Synchronisation is the process of synchronising the generator terminal voltage(s) with an existing distribution grid 
in order to make an electric connection. This process should be executed as such that the least amount of disturbance is caused to both, the generator and the grid.

The generator synchronisation conditions are as follows (Chapman 2012):

1. The RMS line voltages of the generator must be equal to the electrical grid. Otherwise, it may cause a large current flow when the switch is closed.

2. The generator must have the same phase sequence as the grid; in order to avoid different current flow between the phases.

3. The phase angles of the generator and the electrical grid phases must be equal.

4. The generator frequency must be slightly higher than the nominal frequency of the electrical grid. This is to prevent the generator from consuming power instead of supplying power, during frequency stabilisation. If the frequencies are not nearly equal, it will cause large power transients to the generator during the connection process until it stabilises at a common frequency.

In order to achieve the synchronisation process successfully a hydro power controller needs to engage the turbine governing and generator governing functions in a well planned sequence.

\section{Modelling}

All the main controller functions as described in the previous section are implemented in Modelica (Modelica Association 2013) by use of components from the Modelica Standard library (Modelica Association 2019).

The controller model is split up into separate submodels for each of the sub-controllers function:

- Synchronisation Control

- Frequency Control

- Water-level Control

- Excitation System Controller

Those sub-models are then combined to make up the complete hydro power controller as shown in Figure 3.

Each of these sub-controllers are active during different conditions and during different steps in the synchronisation sequence. An example of this is the frequency controller which is only active before synchronisation, with the objective of bringing the generator up to its nominal speed so that the frequency matches that of the grid. After this has been achieved, the frequency controller is deactivated and the water-level controller is activated which ensures maximum power is produced.

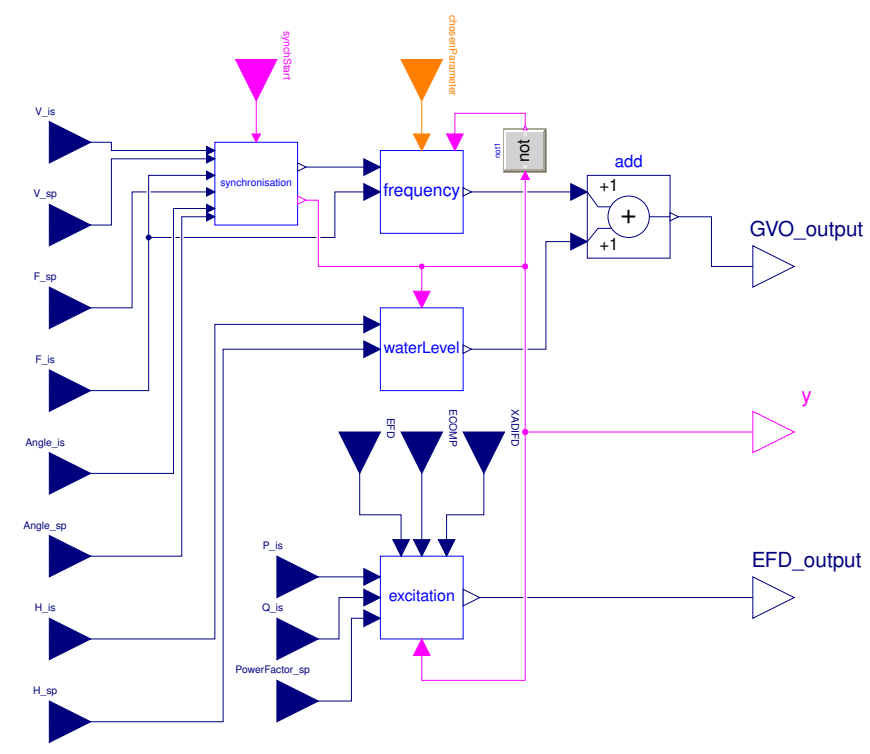

Figure 3. Implementation of the hydro power controller in Modelica

\subsection{Synchronisation Control}

The model of the synchronisation control is shown in Figure 4.

The synchronisation control decides when to close the circuit breaker and to do this it takes in 6 measurement inputs ( $V_{\text {grid }}, V_{\text {gen }}, f_{\text {grid }}, f_{\text {gen }}$, angle grid $_{\text {and }}$ angle gen $\left._{\text {nell }}\right)$ as well as a true/false signal that tells the controller when to allow synchronisation. The controller checks if the measurements are within a set range of each other and if they are, the corresponding block sends a true signal which activates the next block. The sequence of checks are as follows:

\section{Voltage \\ 2. Frequency \\ 3. Angle \\ 4. Phase sequence}

Normally, the synchronisation would only be permitted when all of the above measurements are within the allowed range. However, in the developed model the last requirement (phase sequence) is ignored because it is assumed to be the same for the generator and the grid at all times.

The synchronisation controller will also increase the frequency set-point with a configured offset. This is to ensure that the generator operates at a slightly higher speed than the grid. The reason behind this is that when the grid and generator synchronises, the generator (operating at a higher speed) will be slowed down causing a sudden burst of energy to flow into the grid. This is preferable to the alternative where the grid has a slightly higher frequency than the generator, as this would cause the generator to act 


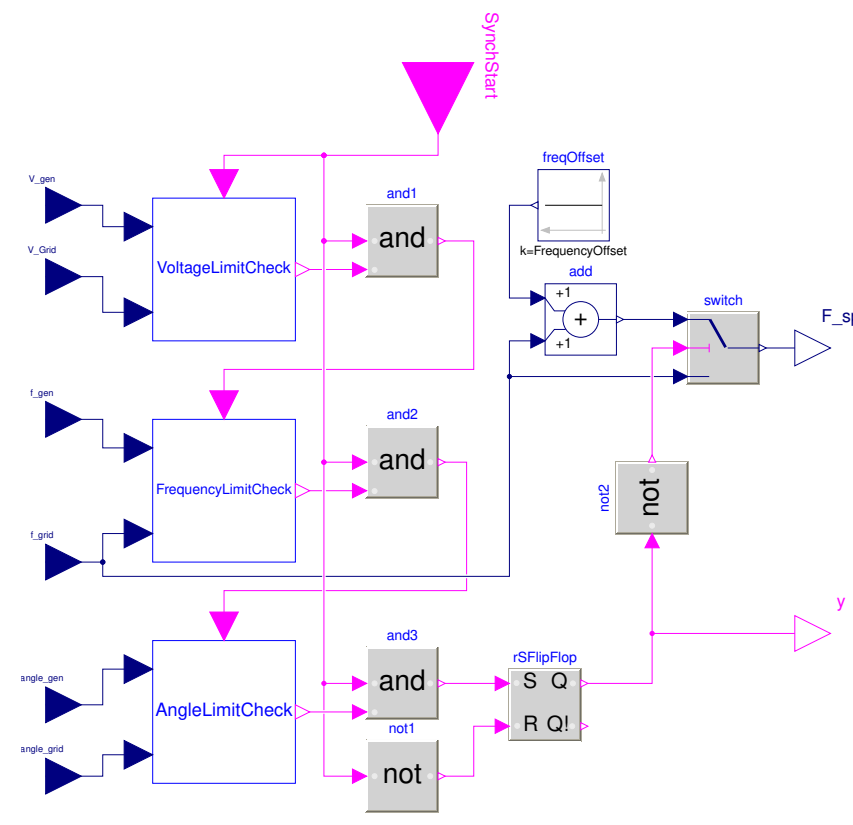

Figure 4. Synchronisation controller in Modelica

like a motor for a short moment. It is important to note that this difference should be kept at a minimum, most preferable zero, but that a slightly higher generator frequency is better than a lower one (Chapman 2012).

\subsection{Frequency Control}

The frequency controller (often also called speed controller) is based on a PID Structure with some added functionality, see Figure 5. The frequency controller has four different sets of PID parameters, each active during different operational modes:

- Disconnected

- Normal

- Unstable

- Isolated

These four parameter sets are implemented in Modelica using four different sets of component, giving four different outputs. Depending on the given integer input the correct output is selected and is used as the output for the frequency controller.

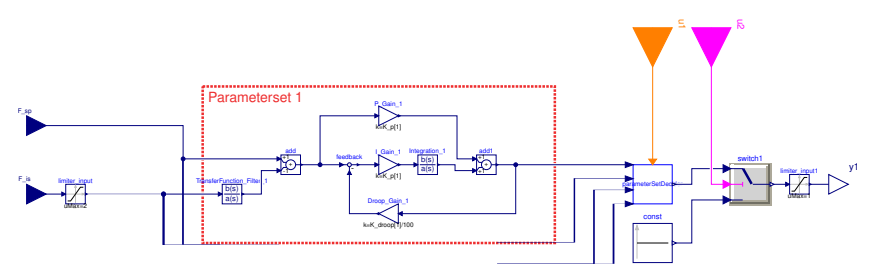

Figure 5. Frequency controller in Modelica

\subsection{Water-level Control}

The water-level controller implemented in Modelica is shown in Figure 6. The purpose of this controller is to allow the hydro power plant to produce as much electrical power as possible when there is enough water. This means that if the water-level falls below a certain set-point, defined in the controller, then the controller will decrease the guide-vane opening of the turbine and thus the mechanical power. One of the requirements of this controller is that the generator is already synchronised to the grid as the frequency needs to be stabilised before the water-level controller is active.

The characteristic of the guide vane opening with respect to the water-level can be adjusted via the look-up table WaterLevelLinearization. Depending on the operator the strategy of how much power (i.e., guide vane opening) should be produced can be different. One strategy can be that one runs the turbine with full guide vane opening as soon as the water-level is above the medium set-point. Others might prefer a more linear dependency as shown in Figure 7.

\subsection{Excitation System Controller}

The excitation system controller can consist of many functions that will affect the voltage reference, such as (IEEE 2016):

- Power system stabilizer (PSS)

- Reactive compensation

- Active compensation

- Frequency droop

- Limiters

$$
\begin{aligned}
& \text { - OEL - Overexcitation Limiter } \\
& \text { - UEL - Underexcitation Limiter } \\
& \text { - SCL - Stator current limiter }
\end{aligned}
$$

- VAR regulator and PF regulator

The purpose of the excitation system controller is to regulate the terminal voltage to different levels that will satisfy various generator operations and system stability conditions. The excitation system controller used in this work is based on the standard static excitation system model ST1A from IEEE 412.5 (IEEE 2016). The ST1A is a potential-source controlled-rectifier excitation system. The excitation system is getting its excitation power supplied through a transformer that is connected to the generator terminals. It contains a PI voltage regulator that compares the generator terminal voltage against the voltage reference, a forward path transient gain reduction, and a feedback loop which acts a stabilising effect on the the excitation. 


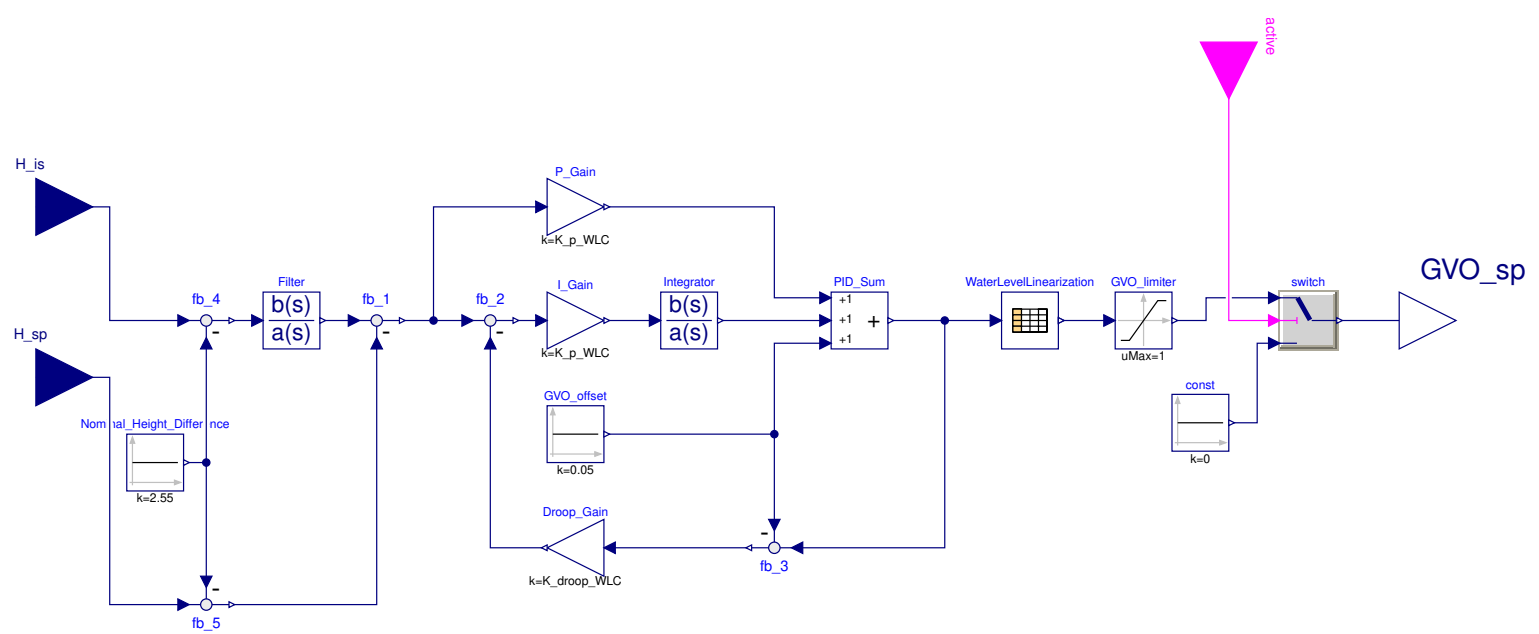

Figure 6. Final implementation of the water-level controller in Modelica

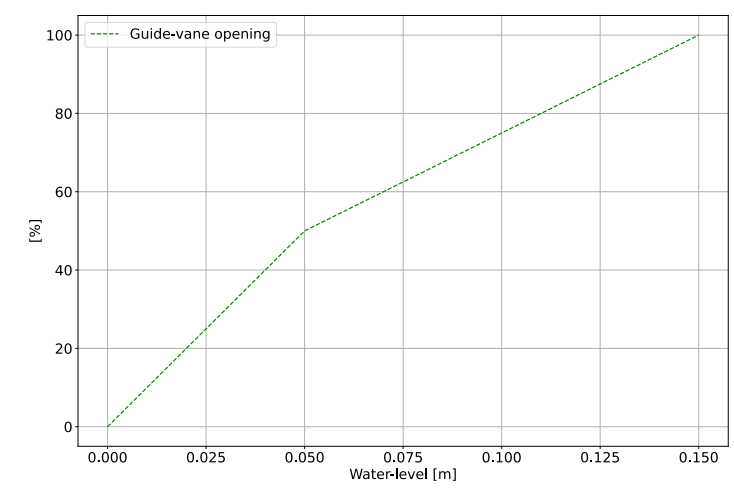

Figure 7. Non-linearity of water-level

As shown in Figure 8 the block diagram of the ST1A model has several inputs like the generator terminal voltage $V_{C}$, the voltage reference $V_{\text {ref }}$, the overexcitation limiter $V_{\mathrm{OEL}}$, the generator field current $I_{\mathrm{FD}}$, and alternative input points for the Power system stabiliser $V_{S}$ and the underexcitation limiter $V_{\mathrm{UEL}}$ (IEEE 2016).

This paper is limited to only include power factor regulator function. Therefore the limiters and stabilisers are disconnected.

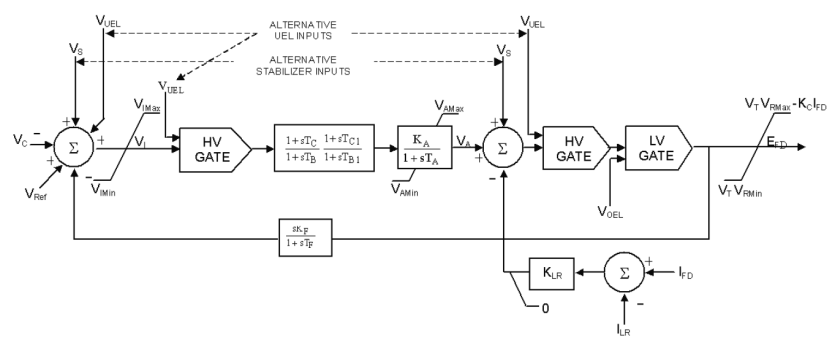

Figure 8. Block diagram of excitation system ST1A from IEEE 421.5-2005 (IEEE 2016)

The STIA excitation system controller is available in the OpenIPSL (ALSETLab et al. 2018) as a PSS®E verified model. A block diagram of the developed excitation system is shown in Figure 9.

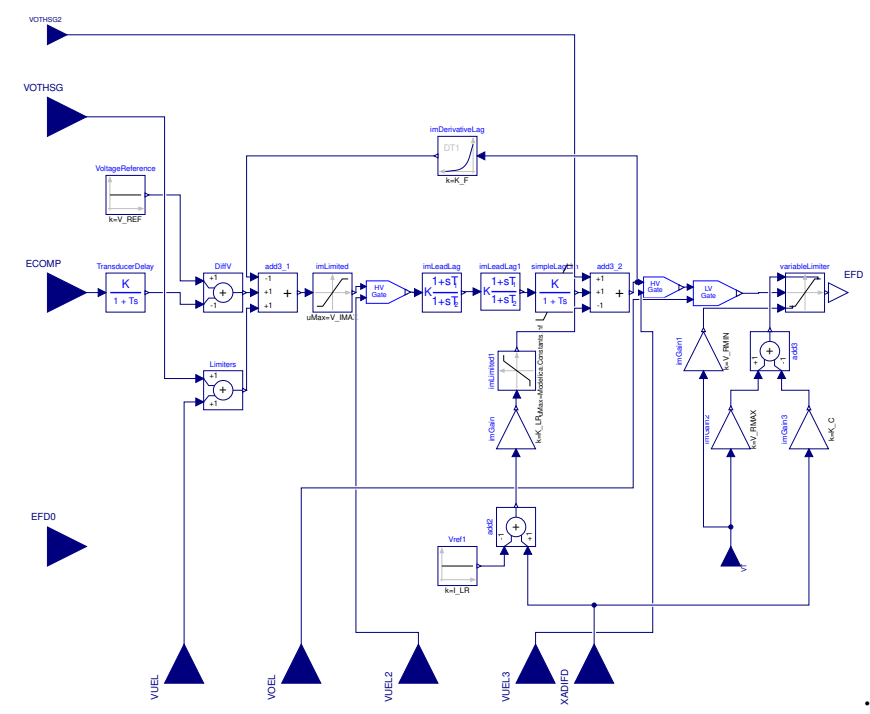

Figure 9. Final implementation of the excitation system ST1A developed in OpenIPSL

\subsubsection{Power Factor Regulator}

The power factor regulator is modelled similar to a voltage regulator, but the voltage input is replaced with a active power $P$ input and reactive power $Q$ input. The power factor regulator shown in Figure 10 is a simplified model based on the standard power factor controller type 2 from IEEE 421.5 (IEEE 2016).

The power factor regulator consists of a power factor normalizer and a PI regulator. It controls the excitation voltage based on whether the generator is operating with a leading power factor or a lagging power factor, i.e., the generator is operating in the overexcited or underexcited region, respectively.

The relationship between variation of excitation field and power factor are displayed in Figure 11 (a). In or- 
der for the power factor regulator to recognise whether the generator is operating in overexcited- or underexcited region, and to deliver the proper control action, the power factor curve needs to be modified to a normalised definition of the power factor. This can be achieved with the following approach shown in Figure 11 (c). Also, this approach will ensure that the normalised power factor is a continuous function as shown in Figure 11 (b).

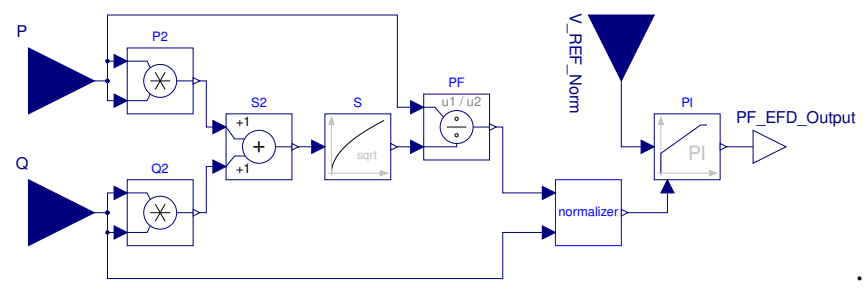

Figure 10. Model of pf controller type 2(IEEE 2016)

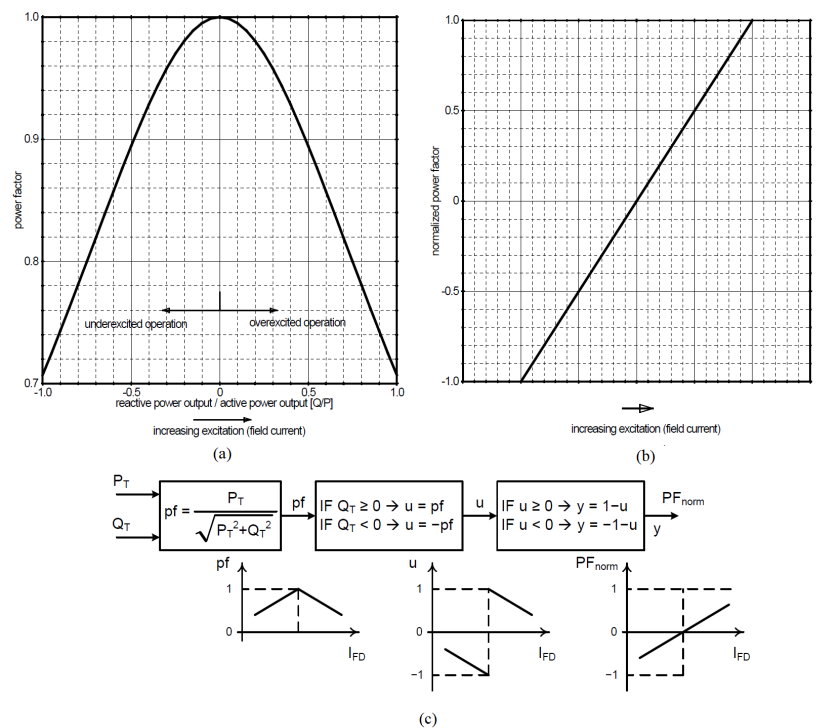

Figure 11. (a) power factor of a generator curve with varying excitation field, (b) normalised power factor from -1 to +1 , (c) model logic scheme for normalised power factor from -1 to +1 (IEEE 2016)

\section{Simulation Results}

\subsection{The Test-Bed model}

The main test-bed model used in order to simulate and verify the implemented model is shown in Figure 12.

It contains the generator model GENSAL from the OpenIPSL, a small simplified grid with an infinite bus to connect to, the hydro power controller as described in section 3 and a model of a real waterway.

The waterway used is a model of a typical small hydro power system in Norway and was created using OpenHPL (TMCC 2019) and is shown in Figure 13. The hydro power plant is run-of-the-river type, where no upstream impoundment is built for water storage. The usable gross-head is about $60 \mathrm{~m}$ water column. The plant consists

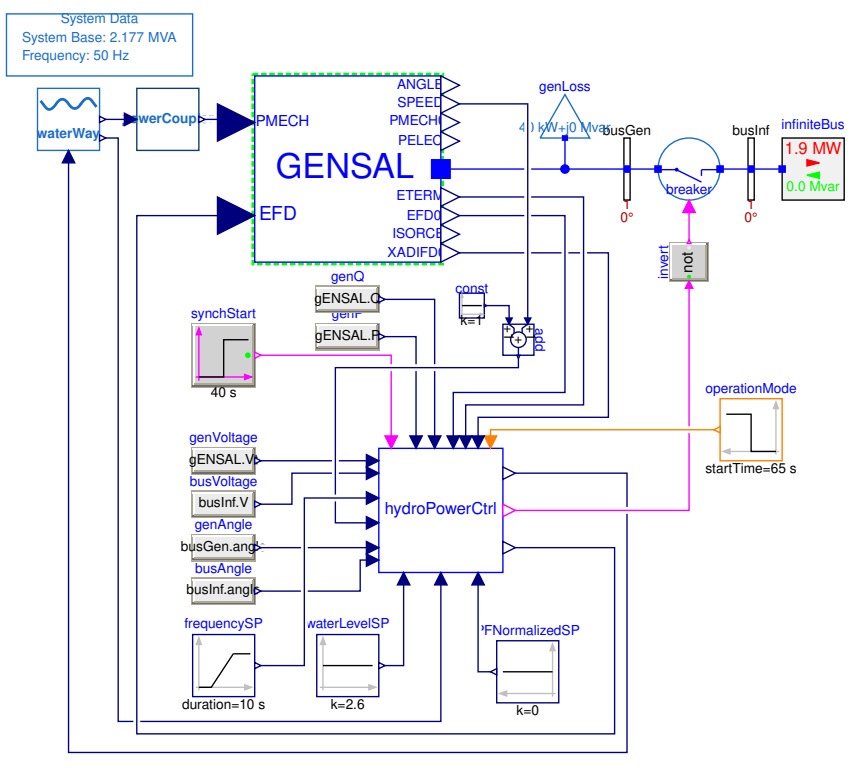

Figure 12. Test-bed model in Modelica

of a $2 M W$ Francis turbine connected to a $2.2 M V A$ synchronous generator.

The test-bed model is using real water-level time series data stored in CSV format which then was read directly using the ModelicaTableAdditions library (Beutlich and Winkler 2021).

\subsection{Synchronisation}

The first action that the hydro power controller will take is to bring the generator from standstill up to the nominal speed. In order to simulate the gradual ramp-up that would occur in the real power-plant, the frequency set-point is changed using a ramp, which starts ramping up at $10 \mathrm{sec}-$ onds. The result is a nice and smooth acceleration up to the nominal set-point, which can be seen in Figure 14.

The large step in the guide-vane opening comes from the fact that the water-level control will take over after the synchronisation in order to produce the maximum power possible. The generator frequency is locked to the infinite bus. Therefore an increase in guide-vane opening will not affect the speed of the generator, but will result in an increase in generator active power output.

During synchronisation the generator will try to reach a slightly higher speed than the grid is operating at which is the reason for the peak in active power at around 50 seconds. This is because when the grid and generator is synchronised, the generator will decelerate and therefore deliver a peak of power to the grid.

The turbine is accelerating up to the nominal speed, the voltage controller will control the excitation voltage of the generator and will adjust the terminal voltage of the generator to be close to equal the voltage of the grid as seen in Figure 15. After the synchronisation process is completed and the circuit breaker is closed, the generator terminal voltage will be locked to the grid, and the power factor controller will become active, aiming to keep the 


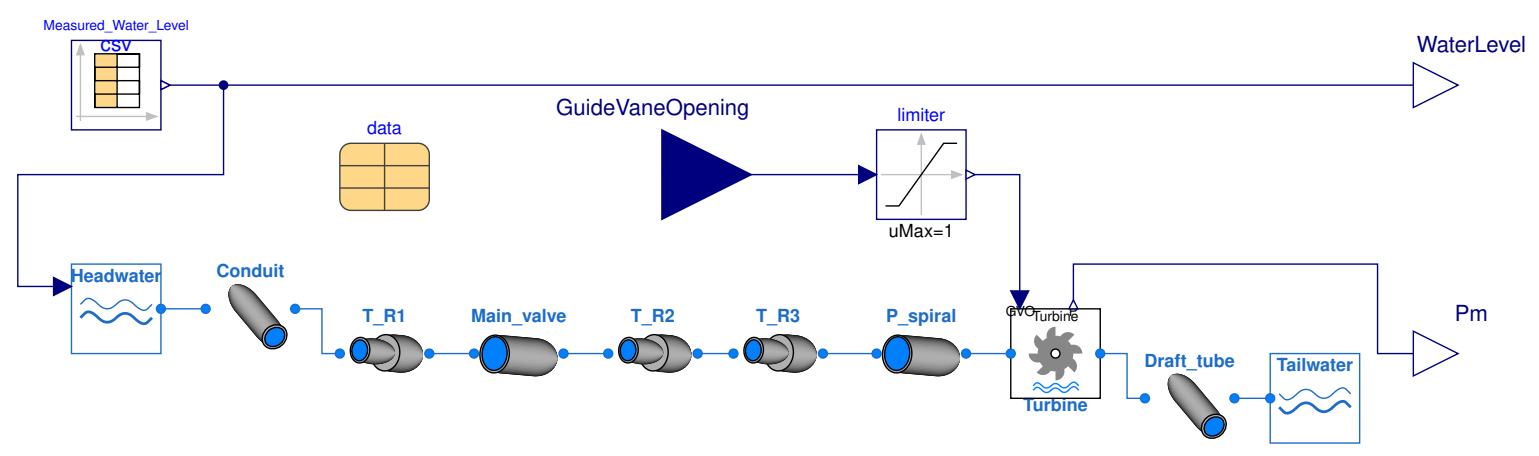

Figure 13. Model of the water way using OpenHPL (TMCC 2019)
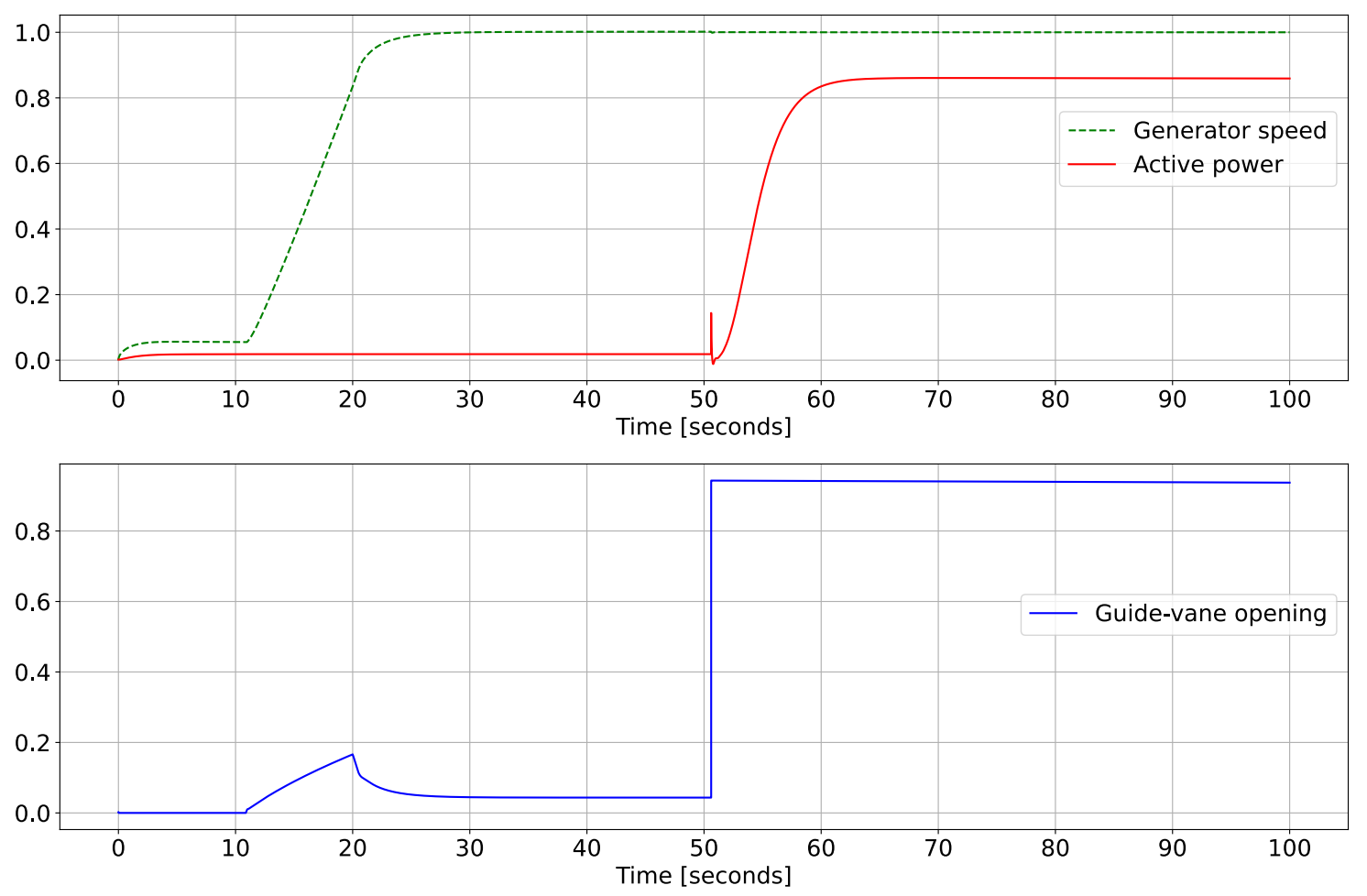

Figure 14. Frequency controller during synchronisation

generated reactive power close to zero.

When the circuit breaker closes at around $t=52 \mathrm{sec}$, there is a slight reactive power peak that is being pushed to the grid. This is because of the generator terminal voltage being slightly higher, and not exactly equal to the grid. The power factor controller reacts to this reactive power peak with a dip in the excitation field (EFD) output.

When the active power production of the generator increases, the generator will start to consume a small amount of reactive power as a response. Therefore, we can see in Figure 15 that the voltage controller due to the power factor controller's influence, will increase the excitation output to compensate for the active power increase, and thus regulate the reactive power to close to zero, making the generator operate at power factor 1 . Considering that the terminal voltage is locked to the grid, and the active power is fixed to mechanical power, an EFD increase will only affect the reactive power.

As mentioned in section 3, there are three conditions before synchronisation is allowed. These are:

- Voltage within limits

- Frequency within limits

- Angle within limits

Before the synchronisation is completed, the frequency set-point is set a bit higher than that of the grid. This offset between the grid and the generator is set to $0.02 \mathrm{pu}$ which is close to $1 \mathrm{~Hz}$, and when the generator frequency is within the chosen limits, a true signal is given, activating the block that compares the generator angle to the grid angle. This frequency behaviour can be seen in Figure 16 and the angle behaviour in Figure 17. 

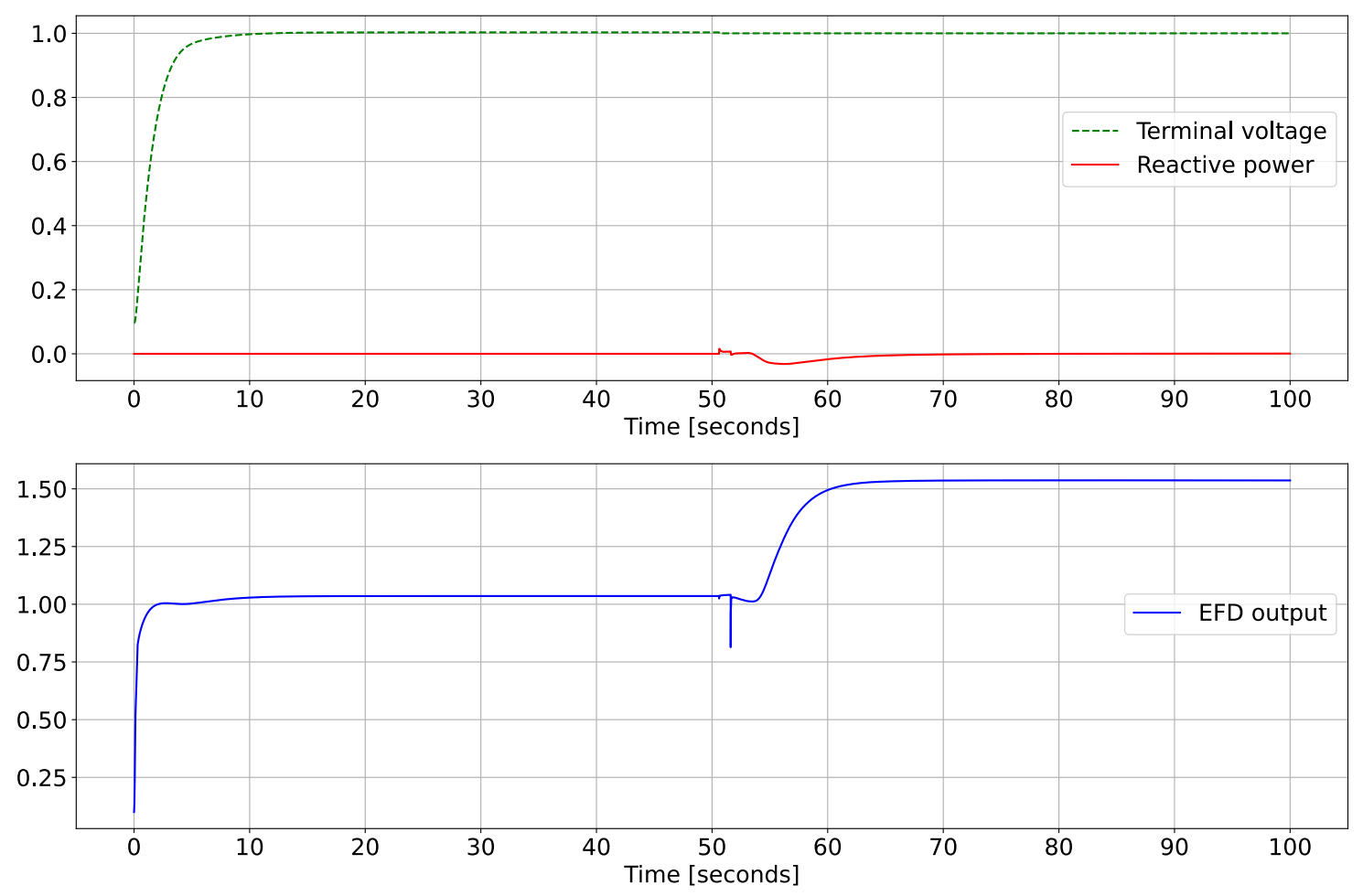

Figure 15. Voltage controller during synchronisation

During the synchronisation it is important that the voltage angle of the generator is leading the bus angle. Otherwise the generator will start as a motor and start consuming power.

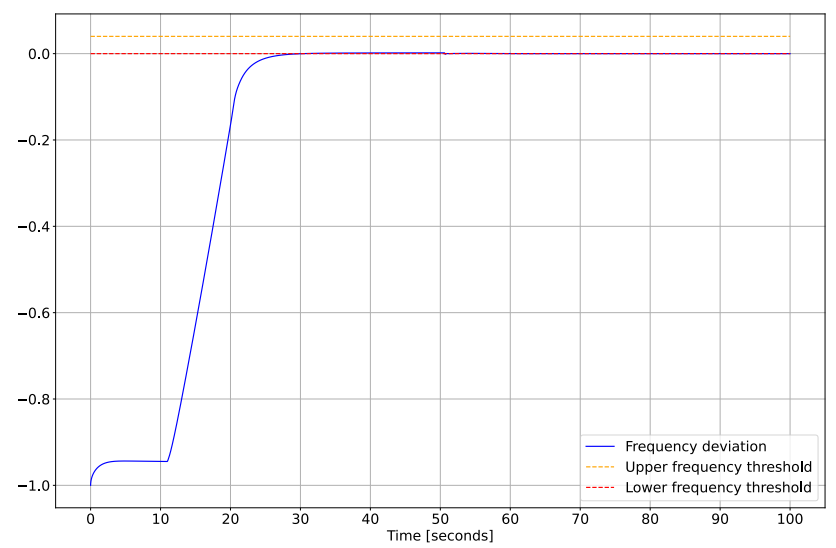

Figure 16. Frequency with the chosen limits

The resulting Boolean sequence of the synchronisation process is shown in Figure 18. Here a true signal is presented on "initiate synchronisation" only when each of the three variables are within the chosen limits (the angle is not shown). During synchronisation when the generator is slowed from the slightly higher frequency down to the nominal frequency of the grid, the frequency will dip slightly below the limits and will thus be false for a fraction of a second. This behaviour is what can be seen in the

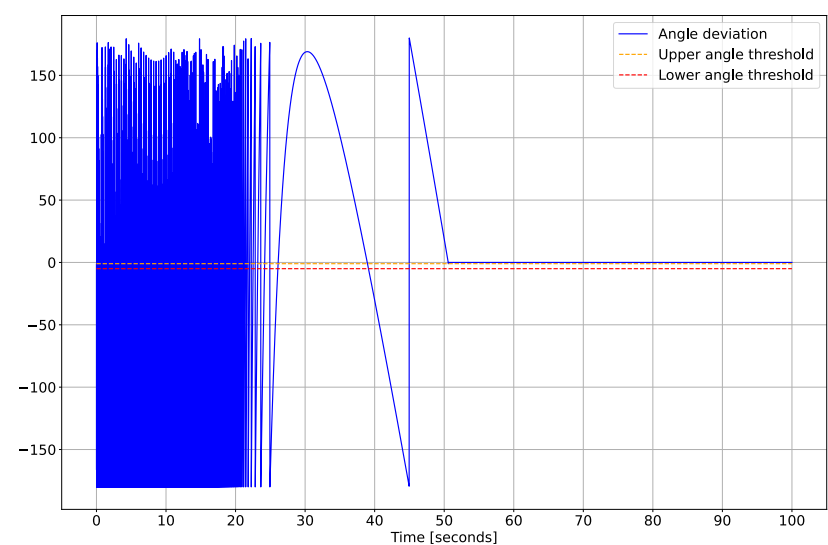

Figure 17. Angle with the chosen limits

middle graph of Figure 18 but as soon as the last check turns true, the circuit breaker is locked so that a sudden frequency dip will not cause the system to fall out of synchronism again.

\section{Discussion}

One of the assumptions made during the modelling of the synchronisation sequence is that the phase sequence for the generator is the same as for the grid. While this is not necessarily true, it is an reasonable assumption. Because of the simplicity of the InfiniteBus component used to simulate the overarching grid, which provides no means of measuring the phase sequence, adding the functionality 

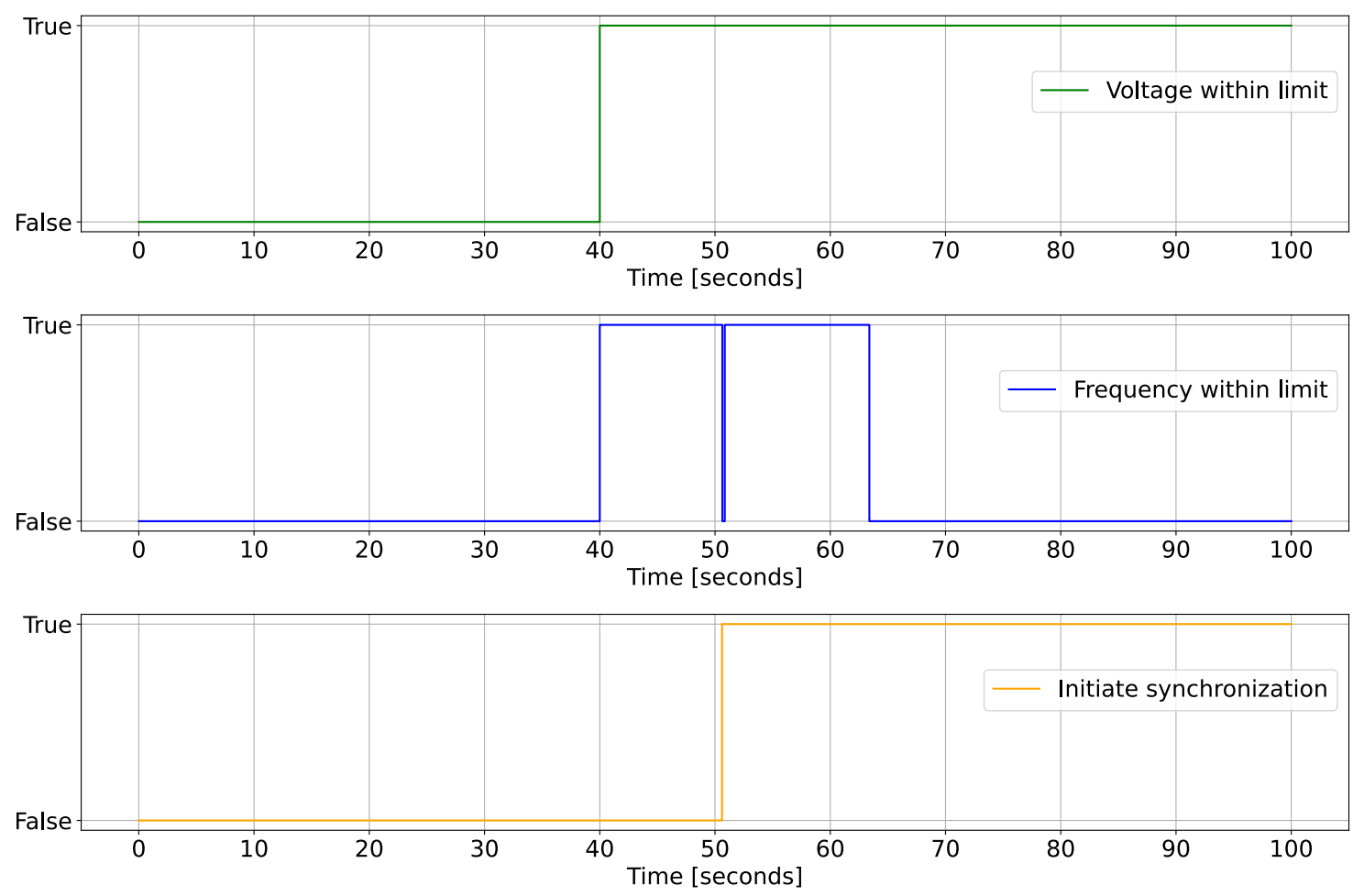

Figure 18. Synchronisation sequence

to the controller would be pointless as there is no way to verify it.

One of the bigger problems during the project was when it comes to verification is that for a lot of these values there was no real measurements available which means that these could not be verified. However, because most of these values still behaved realistically it was decided that this was good enough but that one of the goal with any future work is to attempt to get access to more measurements and verify the simulated values.

While some documentation regarding the different functions of the controllers was available som parameters had to be estimated. This means that for some of the functions, mainly voltage controller, a simulated version of the real controller could not be achieved as not enough information on the exact internal design was available.

\section{Conclusions}

Based on the documentation and parameter values received from power system operators and manufacturers, a model of the turbine controller was created using the Modelica Standard Library, the OpenIPSL and the OpenHPL.

During modelling, the finalised controller was divided up in to several sub-controllers. These were modelled individually and then fitted together into the finished model. The main controllers developed are the controllers for the water-level, frequency and power factor and these were then compared to the theoretical behaviour of these types of controller.
Overall the model functions and behaves as desired, but still needs proper tuning and further development.

\section{Further Work}

Some of functions typically present in a hydro power controller and outlined in section 2 are still missing. Future projects will continue to improve the model and also gather more data for verification. This also includes the grid model that will be replaced with a more complex model including station transformers and distribution grid components.

A continuation of this project has already been successfully completed and the results can be found in the paper "Developing Protective Limiters for a Hydro Power Controller in Modelica" (Manoranjan and Winkler 2021).

\section{References}

ALSETLab et al. (2018). "OpenIPSL: Open-Instance Power System Library - Update 1.5 to "iTesla Power Systems Library (iPSL): A Modelica Library for Phasor Time-Domain Simulations"', In: SoftwareX 7, pp. 34-36. ISSN: 23527110. DOI: 10.1016/j.softx.2018.01.002.

Beutlich, Thomas and Dietmar Winkler (2021). "Efficient Parameterization of Modelica Models". In: Proceedings of the 14th International Modelica Conference. 14th International Modelica Conference. Linköping Electronic Conference Proceedings. Linköping, Sweden: Linköping University Electronic Press, Linköpings universitet. URL: https://github.com/ tbeu/ModelicaTableAdditions. 
Chapman, Stephen J. (2012). Electric Machinery Fundamentals. 5th ed. New York: McGraw-Hill Higher Education. ISBN: 978-0-07-132581-3.

IEEE (2016). 421.5-2016 - IEEE Recommended Practice for Excitation System Models for Power System Stability Studies. IEEE. DOI: 10.1109/IEEESTD.2016.7553421.

Manoranjan, Luxshan and Dietmar Winkler (2021). "Developing Protective Limiters for a Hydro Power Controller in Modelica". In: Proceedings of the 14th International Modelica Conference. 14th International Modelica Conference. Linköping Electronic Conference Proceedings. Linköping, Sweden: Linköping University Electronic Press, Linköpings universitet.

Modelica Association (2013). Modelica - a Unified ObjectOriented Language for Systems Modeling. Language Specification Version 3.2 Revision 2. Linköping: Modelica Association. URL: https : / / www . modelica . org / documents / ModelicaSpec32Revision2.pdf.

Modelica Association (2019). Modelica Standard Library. Version 3.2.3. URL: https : / / github . com / modelica / ModelicaStandardLibrary.

OD Thapar (2008). Modern Hydroelectric Engineering Practice in India: Electro-Mechanical Works. Vol. 1. Department of Hydro and Renewable Energy. URL: https://www.iitr.ac.in/ departments/HRE/uploads/modern_hydroelectric_engg/vol_ 1/Chapter-6_Hydro-Turbine_Governing_System.pdf.

Petroleum, Ministry of and Energy (2016). Renewable Energy Production in Norway. URL: https://www.regjeringen.no/ en / topics / energy / renewable - energy / renewable - energy production-in-norway/id2343462/ (visited on 2020-08-31).

Pterra Consulting (2021). Approaches to Complying with NERC Standard PRC-019-2 on the "Coordination of Generating Unit or Plant Capabilities, Voltage Regulating Controls, and Protection". URL: https : / / www . pterraph . com / nerc compliance/approaches-to-complying-with-nerc-standardprc-019-2-on-the-coordination-of-generating-unit-or-plantcapabilities-voltage-regulating-controls-and-protection/ (visited on 2020-11-16).

Statkraft (2020). Hydropower. URL: https://www.statkraft.com/ what-we-do/hydropower/ (visited on 2020-08-31).

TMCC (2019). OpenHPL: Open Hydro Power Library. Version 1.0.0. University of South-Eastern Norway. URL: https: //openhpl.simulati.no. 